\title{
How to read easily
}

Charles Jones

George Mason University

cjones@gmu.edu

The function of the semi-obligatory adverbial element in English-type middles, e.g., often, easily, has received various kinds of treatments, often in the service of various implicit elements considered to be characteristic of the construction. For example, Hoekstra \& Roberts 1993 propose that it provides an Experiencer argument to license the implicit Agent argument. Condoravdi 1989 proposes that it supplies the semantic content for the nuclear scope of an implicit generic quantificational operator. In other places, there is relative silence; e.g., Stroik's work (2006). In this paper I outline and discuss a somewhat novel reading of the adverbial element in which it relates directly only to the overt elements of the construction, as in (1). Particularly, it must relate to subject NP.

$$
\text { [NP this bread] [vp [v cuts] easily] }
$$

\section{A reading}

We wish to find a reading of the middle adverbial that is predicative of the subject NP. The subject orientation of the adverbial can be clearly seen in PP-mediated middles like (2), where [pP like real meat] is a transparent candidate for a predicate of this dog food.

With respect to adverbial easily in (1), a subject-NP predicate reading of it is suggested by the non-middle (3), where easily characterizes the conductive property of the wire.

This wire conducts (electricity) easily.

The conductive property of the wire is a property of the wire itself - electricity neither causes nor determines it. In (3) the conduct VP contributes to the context in which easily is to be interpreted. With respect to electrical conductivity, and quite generally, easily is interpreted as something like with low resistance. In like manner, the easily of (1) can be read as a characterization of some properties of the bread, relative to being cut. Abstracting from tense, a simple semantic representation for $(1)(=(4 a))$ that reflects such a reading would be $(4 b)$, paraphrased as in $(4 c)$.

(4) a. This bread cuts easily.

b. $\quad \lambda e[\operatorname{CUT}(b)(e) \& \operatorname{EASY}(b)(e)]$

c. "In an event of being cut, this bread offers little resistance (this bread is 'easy')."

In short, (4b) represents, and (4c) paraphrases, how to read easily.

\section{Composition}

Maintaining our focus on the simple overt elements of the middle, we outline here what we want them to do to compose a workable syntax that will yield the semantics of (4b). 


\subsection{Properties and functions}

A semantic framework that directly characterizes what we want from the middle constituents is a property theory like that in Chierchia 2004 (henceforth, property theory). In property theory, there are three kinds of primitives: entities $e$, propositions $p$, and properties $\pi$. Properties lead a kind of double life, as primitives $\pi$ and as propositional functions $<e, p>$. The conversion of properties to functions is mediated by some operator, call it $\Pi$. We are interested in the syntactic effect of $\Pi$, which sets up a non-trivial non-sister Spec position for an element X of type $\pi$.

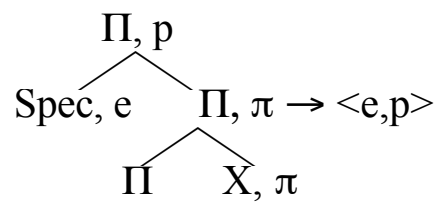

This Spec provides a syntactic position for the $e$ argument of the the $<e, p>$ propositional function, (along purely configurational lines, as outlined in Hale \& Keyser 2002). ${ }^{1}$

A good approximation, which we assume here, of the kind of verb that allows the middle construction is "change-of-state". Suppose, as is common, that the logical representation of a change-of-state verb like cut is simply an abstract predicate of its object argument, signifying its resultant state, as in (6). ${ }^{2}$

$$
\text { Change of state: } c u t: \quad \lambda x \lambda \mathrm{e}[\operatorname{CUT}(\mathrm{x})(\mathrm{e})]
$$

Abstract CUT in (6) is a property $\pi$. Let us call this abstract property the object property.

\subsection{Derivation}

Assuming an easily-adverbial is a $<\pi, \pi>$ operator, let us now suppose that the object property cut, of the bare V cut, is visible to adverbial adjunction (Kratzer 2000). We can exploit V-level adjunction of the easily-adverbial to generate a Spec position for the assignment of the argument of the embedded abstract object property. In a structure like (7), the argument structure of the central $\mathrm{V}$ is laid out inside VP, and the semantics follow straightforwardly, and simply, as a conjunction of properties to be predicated of the subject argument.

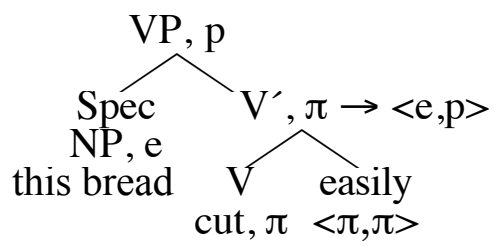

6. $\lambda \mathrm{e}[\mathrm{CUT}(\mathrm{b})(\mathrm{e}) \& \operatorname{EASY}(\mathrm{b})(\mathrm{e})]$

5. this bread $=b$

4. $\lambda x \lambda e[\operatorname{CUT}(x)(e) \& \operatorname{EASY}(x)(e)]$

3. $\lambda \mathbf{P} \lambda \mathrm{x} \lambda \mathrm{e}[\mathbf{P}(\mathrm{x})(\mathrm{e}) \& \operatorname{EASY}(\mathrm{x})(\mathrm{e})](\lambda \mathrm{x} \lambda \mathrm{e}[\mathrm{CUT}(\mathrm{x})(\mathrm{e})])$

2. easily $=\lambda \mathbf{P} \lambda \mathrm{x} \lambda \mathrm{e}[\mathbf{P}(\mathrm{x})(\mathrm{e}) \& \operatorname{EASY}(\mathrm{x})(\mathrm{e})]$

1. $\operatorname{cut}=\lambda \mathrm{x} \lambda \mathrm{e}[\mathrm{CUT}(\mathrm{x})(\mathrm{e})]$

1 Hale \& Keyser 2002:12, (23b): "Specifier-head: If $\mathrm{X}$ is the specifier of a head H, and if $\mathrm{P}_{1}$ is the first projection of $\mathrm{H}\left(\mathrm{H}^{\prime}\right.$, necessarily nonvacuous $)$, then $\mathrm{X}$ is the unique sister of $\mathrm{P}_{1}$."

2 We arrive at (6) by getting rid of the external argument, as well as elements of any structure associated with the semantics of cause (assumed to be associated with functional heads outside VP (Kratzer 1996, Hale \& Keyser 2002)).

If, for some reason, the logical representation of cut requires the external argument, CAUSE, etc., we would in turn require a "rule" of middle formation. Such a rule would begin by eliminating these features, much as various treatments of the middle already do in some fashion, e.g., "Assign arb to the external $\theta$-role" (Fagan 1988: (67)). 
In (7), the adverbial acts as (5)'s predication operator $\Pi$ in setting up the structure necessary for a non-trivial, non-sister to the head V. We take (7) as the core syntax and semantics (7.6) of the English middle. A construction like (7) predicts a couple of interesting characteristics of the middle.

(8) a. The middle is restricted to verbs with embedded abstract properties. This restriction is consonant with the embedded property of "change-of-state" semantic representations.

b. Access to the abstract property of the middle is via $<\pi, \pi>$ adverbial adjunction, which in general requires no special morphology. The English middle conspicuously lacks special verbal morphology.

\section{Extras}

\subsection{Agent}

There is no mention of an extra participant in (7.6), as the properties denoted by the VP are predicated of the subject only. These properties are independent of any Agent of the cutting, or of any Experiencer of it, as would be required by Hoekstra \& Roberts' 1993 licensing scheme. There, an (implicit) Experiencer of the ease (of the killing) must license the (implicit) Agent of the killing, as in (9).

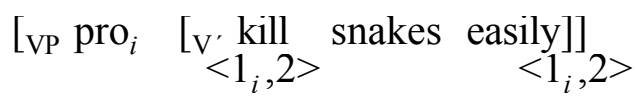

In the present scheme, it is simply the snakes that are "easy" (= offering little resistance to the killing). Restricting easily to the subject of the object property, rather than allowing it to associate with any other implicit argument, begins to make sense of the well-known inability of Agentoriented adverbials to license the middle: *This bread cuts carefully.

As is also well-known, an NP providing much of the thematic content of "Agent" in a middle can be syntactically introduced via a for-PP (Stroik 2006), as in (10).

This bread cuts easily [PP for Bill]

There is nothing to preclude the introduction of a new argument into the semantics (Kratzer 1996). ${ }^{3}$ However, there is nothing in the object property predication (7.6), complete as it is, that requires it. Syntactically and semantically, the for-PP can only be an adjunct.

\subsection{Genericity}

While the core middle predication in (7.6) has no expression of genericity, the raw material, i.e., an event variable, is there. Condoravdi 1989 makes the adverbial element an essential part of the middle by having it as the nuclear scope of a generic operator.

(11) a. This bread cut smoothly.

b. $\mathrm{G}[\mathrm{e}: \operatorname{bread}(\mathrm{x})$, cut $(\mathrm{e})$, Patient $(\mathrm{e}, \mathrm{x})][\operatorname{smooth}(\mathrm{e})] \quad \mathrm{C}: 18,(5)$

3 Bill would be introduced via some thematic predicate $\theta$, associated with for, as in $\lambda \mathrm{x} \lambda \mathrm{e}[\theta(\mathrm{x})(\mathrm{e})]$, and its event argument would be identified with that of the matrix predicate via Event Identification (Kratzer 1996).. 
Condoravdi's partition of the predicates in (11b) has it that, generally, events of cutting the bread go smoothly. What is missing from (11) is some connection between the smoothness associated with the event and the bread itself, which is, after all, the (denotation of the) subject of the middle (11a). In the present scheme, it is a property of the bread, "smooth" (= with low resistance), that makes the [cut smoothly] predicate true. This kind of more direct connection of the predicate with the subject is rendered, for example, in a generic analysis along the lines of Chierchia 1995.

(12) a. Gen e $[\operatorname{IN}(b)(e)][\operatorname{CUT}(b)(e) \& \operatorname{EASY}(b)(e)]$

b. "Generally, when this (kind of) bread is around, it (has the property:) cuts easily."

At the heart of (12a) is the same kind of simple predication as in (7.6): [easy $(x)(e)]$ ' $x$ is easy'. In general, it is this core predicate that must be true in any instance that makes the generalization true. This is the "bottom line" for the semantics of the middle.

(13) Bottom line semantics for the middle

The properties composed by the object property of middle $\mathrm{V}$ and its adverbial are predicated of the subject, in any situation or possible world, or with any associated participant.

Assuming (13), non-generic middles, with definite times and subjects, should be unremarkable.

(14) a. (After it thawed,) this (particular) steak cut easily.

b. PAST $\exists$ e $[\operatorname{CUT}(\mathrm{s})(\mathrm{e}) \& \operatorname{EASY}(\mathrm{s})(\mathrm{e})]$

\section{References}

Chierchia, Gennaro. 1995. Individual-level predicates as inherent generics. Carlson \& Pelletier, eds. The generic book. Chicago: University of Chicago Press, 176-223.

Chierchia, Gennaro. 1989/2004. A semantics for unaccusatives and its syntactic consequences. Alexiandou, Anagnostopoulou, \& Everaert, eds. The unaccusativity puzzle. Oxford: Oxford University Press, 22-59.

Condoravdi, Cleo. 1989. The middle: where semantics and morphology meet. MITWPL 11: 18-30.

Fagan, Sarah M. B. 1988. The English middle. Linguistic Inquiry 19:181-203.

Fellbaum, Christiane. 1985. Adverbs in agentless actives and passives. In Eilfort, Kroeber \& Peterson (eds.) CLS 21.2: Papers from the parasession on causatives and agentivity. University of Chicago: CLS, 21-31.

Hale, Kenneth L. \& Samuel J. Keyser. 2002. Prolegomenon to a theory of argument structure. Cambridge, MA: MIT Press.

Hoekstra, Teun \& Ian Roberts. 1993. Middle constructions in Dutch and English. Reuland \& Abraham (eds.), Knowledge and Language II. Dordrecht: Kluwer, 183-220.

Kratzer, Angelika. 1996. Severing the external argument from its verb. Rooryck \& Zaring, eds. Phrase structure and the Lexicon: Dordrecht: Kluwer: 109-137.

Kratzer, Angelika. 2000. Building statives. Berkeley Linguistic Society 26.

Stroik, Thomas. 2006. Arguments in middles. In Lyngfelt \& Solstad (eds.), Demoting the Agent. Amsterdam: John Benjamins, 301-326. 